\title{
through Porous Medium Enclosing a Rectangular Isothermal Body
}

\author{
Dr. Amir S. Dawood \\ Mechanical Engineering \\ Department, \\ College of Engineering, Mosul \\ University
}

Osama Basil Hmood, Mechanical Engineering Department, Faculty of Engineering \& Technology Sebha University, BrackAshati, Libya

\section{Abstract:}

Steady free convection through a porous medium around a rectangular isothermal body has been numerically investigated. The isothermal body is kept at constant low temperature and the porous medium has an impermeable rectangular boundaries. The cavity wall of porous material has a constant high temperature.

The full governing equations (momentum an energy equation) have been solved for range of values of the governing parameters by using the finite difference method and covered a wide range of modified Rayleigh number (Ra) (0-500) with different sizes of isothermal body. Results are presented in terms of the streamlines and isotherms to show the behavior of the flow and temperature fields. This study shows that the Nusselt number $(\mathrm{Nu})$ is a strong function of the modified Rayleigh number, the isothermal body size and boundary conditions. For certain range of $\mathrm{Ra}$, the rate of heat transfer decreases when the flow divided into primary and secondary cells.

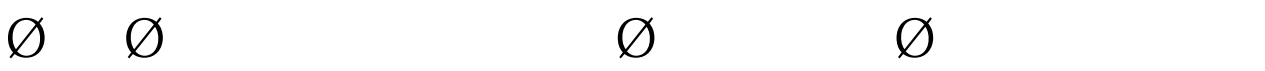

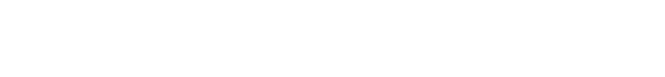

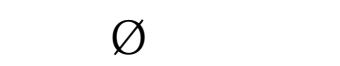

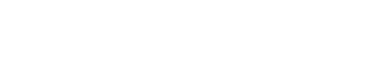

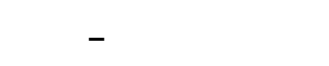

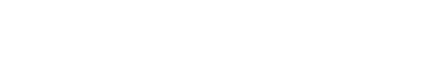

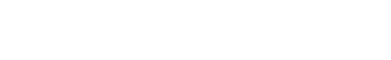

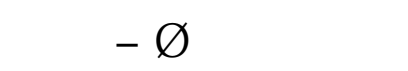

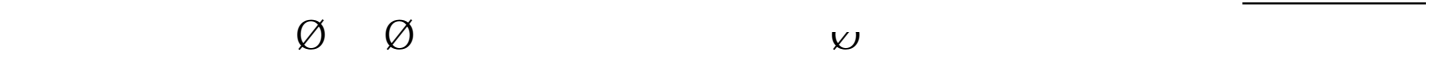 \\ ú̌̄ญ

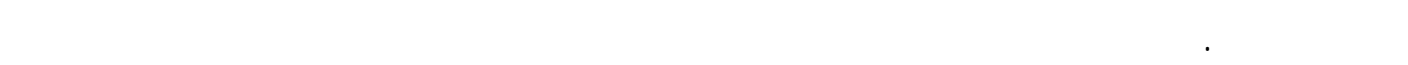

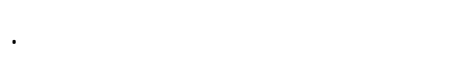

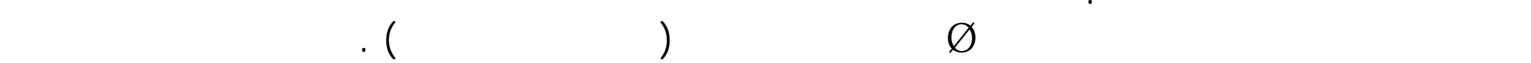

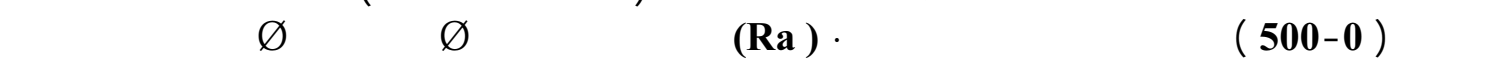

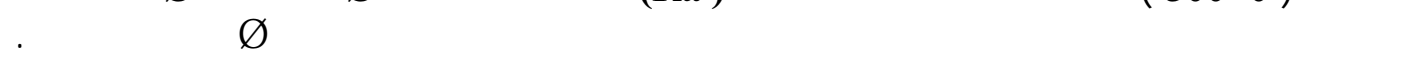

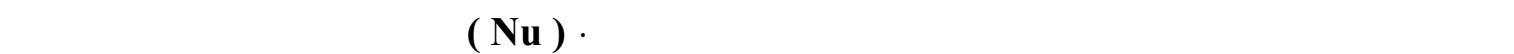

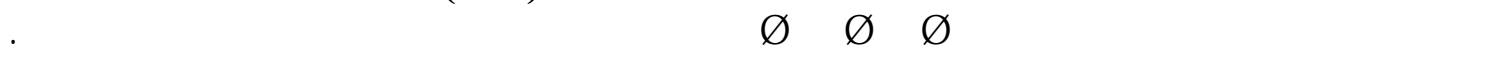

Received 20 ${ }^{\text {th }}$ Aug. $2002 \quad$ Accepted $19^{\text {th }}$ July 2005 


\section{Nomenclature:}

A

$\mathrm{C}_{\mathrm{p}}$

g

$\mathrm{H}$

$\mathrm{K}$

$\mathrm{k}_{\mathrm{e}}$

$\mathrm{L}$

$\mathrm{Nu}$

$\mathrm{P}$

$\mathrm{Ra}$

$\mathrm{T}$

$\Delta \mathrm{T}$

$\mathrm{u}$

$\mathrm{V}$

$\mathrm{x}, \mathrm{y}$

A

$\mathrm{B}$

$\theta$

$\mu$

$\mathrm{P}$

$\psi$

Subscripts

$\mathrm{c}$

$\mathrm{e}$

F

$h$

\section{o}

Superscript

Aspect ratio, $\mathrm{H} / \mathrm{L}$

Specific heat at constant pressure, (J/kg.K)

Acceleration due to gravity, $\left(\mathrm{m} / \mathrm{s}^{2}\right)$

Height of porous cavity, (m)

Permeability of porous medium, $\left(\mathrm{m}^{2}\right)$

Effective thermal conductivity of porous medium, (W/m.K)

Width of porous cavity, (m)

Nusselt number

Pressure, $\mathrm{Pa}$

Modified Rayleigh number

Dimensional temperature, $\mathrm{K}$

Temperature difference, $\mathrm{K}$

Fluid velocity in $\mathrm{x}$ direction, $\mathrm{m} / \mathrm{s}$

Fluid velocity in y direction, $\mathrm{m} / \mathrm{s}$

Cartesiane coordinates

Thermal diffusivity of porous medium, $\left(\mathrm{m}^{2} / \mathrm{s}\right)$

Coefficient of thermal expansion of flluid, $\left(\mathrm{K}^{-1}\right)$

Dimensionless temperature

Dynamic viscosity, $(\mathrm{kg} / \mathrm{m} . \mathrm{s})$

Density of fluid, $\left(\mathrm{kg} / \mathrm{m}^{3}\right)$

Stream function

Cold wall

Effective

\section{Fluid}

Hot wall

Reference condition (for the media)

Dimensionless parameter

\section{Introduction}

The analysis of natural convection heat transfer in a porous medium has been an important subject due to its increasing applications in many industrial and geophysical problems, particularly in oil exploration, chemical and nuclear industries [1]. In the field of energy conservation more attention had been paid to the insulation characteristics of the saturated porous materials introduced in some enclosures (storage tanks) to decrease the convective heat transfer [2].

Many two-dimensional numerical solutions exist in the literature for a rectangular and cylindrical enclosure, filled with a porous medium with the vertical sides subjected to different temperatures. Most of them are directed 
Dawood : Numerical study of free convection heat transfer through porous.....

toward thermal insulation applications of storage tanks, nuclear reactors, and buildings.

In the present work, two-dimensional numerical analysis is presented for natural convection from a rectangular isothermal body embedded in a confined rectangular porous cavity. This configuration occurs in ducts covered by insulating materials. The inner isothermal body is kept cold at constant temperature, while the outer boundaries of the cavity are kept hot at constant temperature as shown in figure (1).

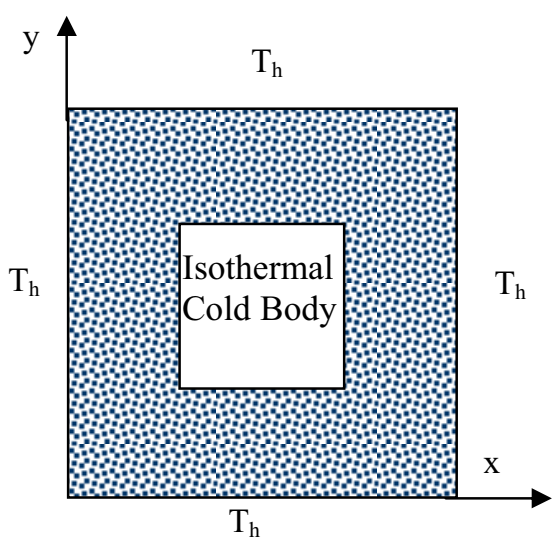

Figure (1) Geometry of the Problem

\section{Literature Review}

A wide variety of situations have been studied previously. These studies were analytical, numerical, experimental or any combination of these. Nobuhiro et al [3] studied experimentally the convective heat transfer in a confined rectangular cavity packed with porous media. They studied two kinds of solid particles with three kinds of fluid, their experiments covered a wide range of Rayleigh number $\left(1-10^{5}\right)$, and aspect ratio (height to width of cavity) from 5 to 26 . The effect of temperature on boundary layer thickness had been explained for different structure of solids and fluids. Prasad and Kulacki [4] studied numerically, using finite difference method, natural convection in a rectangular porous cavity. The effect of aspect ratio was summarized by a family of curves, for aspect ratios from 0.05 to 100 . It had been found that for an aspect ratio less than one a multicelluar flow developed.

Oothuizen [5] studied two dimensional flow over a horizontal hot plate in a saturated porous medium mounted near an impervious adiabatic horizontal surface and subjected to horizontal forced flow, where the usually Darcy model is adopted. This problem was numerically investigated by using the finite element method. He concluded that the heat transfer from the plate

is influenced by the dimensionless depth of the plate below the surface and the importance of the buoyancy forces. 
Arnold [6] studied the natural convection in a porous medium between two concentric, horizontal cylinders. Two dimensional equations had been solved, using a very fine mesh. For radius ratio $(\mathrm{R}=2)$, a steady four-cell regimes were seen to occur at a Rayleigh number of about 120, further increase of the Rayleigh number did not result in the appearance of more cells. However, for $\mathrm{R}=1.2$, a four-cell regime appears at $\mathrm{Ra}=250$ and up to eight cells were observed on increasing the Ra. A similar study of Arnold had been obtained by Barbosa Mota and Saatdjian [7]. An accurate second-order finite difference scheme using an alternative direction method and successive under-relaxation was applied to finer grid as compared with that of Arnold. At radius ratio $(\mathrm{R}=2)$, the flow changes from a two-cell to a four cell regime at $\mathrm{Ra}=110$, similar behavior has been found in this work. Among the previous works, no one had studied the present case.

\section{Governing Equations}

The equations that govern the fluid and heat flow in a saturated porous medium are the continuity, momentum, and energy equations.

3.1. Continuity Equation

The continuity equation for steady two-dimensional incompressible flow in an isotropic porous medium is:

$$
\frac{\partial u}{\partial x}+\frac{\partial v}{\partial y}=0.0
$$

\subsection{Momentum Equation}

In porous medium the momentum equation is derived from Darcy law, which is based on measurement alone. This law states: "the area averaged fluid velocity through a column of porous material is directly proportional to the pressure gradient established along the column, in addition, the velocity is inversely proportional to the viscosity ( $\hat{a}$ ) of the fluid seeping through the porous medium" [8]. Then for two dimensions with the presence of gravitational acceleration the Darcy model is:

$$
\begin{aligned}
& u=-\frac{K}{\mu} \frac{\partial p}{\partial x} \\
& v=-\frac{K}{\mu}\left(\frac{\partial p}{\partial y}+\rho g\right)
\end{aligned}
$$

Where $\mathrm{K}$ is an empirical constant and is called the permeability.

To admit the buoyancy effect the Boussinesq approximation is used. This approximation can be applied to both liquid and gases:

$$
\rho \cong \rho_{\mathrm{O}}\left[1-\beta\left(T-T_{\mathrm{O}}\right)\right]
$$

Using equations (3) and (4) 


$$
v=\frac{-K}{\mu}\left[\frac{\partial p}{\partial y}+\rho_{\mathrm{O}} g\left(1-\beta\left(T-T_{\mathrm{O}}\right)\right)\right]
$$

It is convenient to eliminate the pressure term between equation (2) and (5) by using a cross differentiation and the momentum equation becomes:

$$
\frac{\partial u}{\partial y}-\frac{\partial v}{\partial x}=\frac{-\rho_{\mathrm{O}} g \beta K}{\mu} \frac{\partial T}{\partial x}
$$

\subsection{Energy Equation}

The derivation of energy equation is based on the following assumptions: the medium is homogeneous, isotropic, and the solid matrix is in thermal equilibrium with the fluid filling the pores. These assumptions are adequate for small-pores media such as geothermal reservoir and fibrous insulation. Then for steady-state flow in two-dimensions the energy equation is:

$$
\begin{aligned}
& u \frac{\partial T}{\partial x}+v \frac{\partial T}{\partial y}=\alpha\left[\frac{\partial^{2} T}{\partial x^{2}}+\frac{\partial^{2} T}{\partial y^{2}}\right] \\
& \alpha=\frac{k_{e}}{\rho_{f} C p_{f}}
\end{aligned}
$$

\section{Dimensionless Formulation}

To more conveniently elucidate the mathematical manipulations, the study of the governing equations will be carried out in a non-dimensional form. All the spatial dimensions are non-dimensionalized with respect to $\mathrm{L}$ and $\mathrm{H}$, then

$$
\hat{x}=\frac{x}{L} \quad, \quad \hat{y}=\frac{y}{H}, \quad A=\frac{H}{L}
$$

Where $\mathrm{A}$ is the aspect ratio (height to width). This ratio will remain constant at $\mathrm{A}=1.0$ in the solution.

For temperature

$$
\theta=\frac{T-T_{c}}{T_{h}-T_{c}}
$$

The stream function concept is commonly used for convective heat transfer problem, and is defined in terms of the velocity components, which satisfies the continuity equation:

$$
u=\frac{-\partial \psi}{\partial y}, \quad v=\frac{\partial \psi}{\partial x}
$$

The stream function is non-dimensionalized as follow:

$$
\hat{\psi}=\frac{L}{H \alpha} \psi
$$

Then the governing equations will take the following non-dimensional forms: 


\subsection{Momentum Equation}

$$
A^{2} \frac{\partial^{2} \hat{\psi}}{\partial \hat{x}^{2}}+\frac{\partial^{2} \hat{\psi}}{\partial \hat{y}^{2}}=R a A \frac{\partial \theta}{\partial \hat{x}}
$$

Where $(\mathrm{Ra})$ is a modified Rayleigh number.

$$
R a=\frac{\rho g \beta K L \Delta T}{\mu \alpha}
$$

\subsection{Energy Equation}

$$
\frac{\partial \hat{\psi}}{\partial \hat{x}} \frac{\partial \theta}{\partial \hat{y}}-\frac{\partial \hat{\psi}}{\partial \hat{y}} \frac{\partial \theta}{\partial \hat{x}}=\frac{\partial^{2} \theta}{\partial \hat{x}^{2}}+\frac{1}{A^{2}} \frac{\partial^{2} \theta}{\partial \hat{y}^{2}}
$$

\section{Validation of Numerical Solution}

The validation of numerical solution is accomplished by comparing with the several two-dimensional solutions with an exact solution in the conduction case $(\mathrm{Ra}=0)$. For the conduction case the modeled problem was a rectangular domain subjected to different temperatures on its vertical sides, with the top and bottom insulated. The numerical solution was in good agreement with the exact solution [9].

The present solution is also compared with other previous works of convective heat transfer in a two-dimensional porous cavity, subjected to constant different temperatures on its vertical sides, while the horizontal sides are perfectly insulated.

Table (1) below gives the comparisons at two different values of Rayleigh numbers: 50 and 100 and at aspect ratio of 1 . There are some differences between the present work and others due to finite difference approximation.

\begin{tabular}{|l|l|l|l|l|l|l|}
\hline \multirow{2}{*}{$R a$} & \multicolumn{2}{|c|}{$N u$} \\
\hline \multirow{4}{*}{$\begin{array}{l}50 \\
100\end{array}$} & $\begin{array}{l}\text { Chan et al } \\
(10)\end{array}$ & $\begin{array}{l}\text { Burns et } \\
\text { al }\end{array}$ & $\begin{array}{l}\text { Bejan \& } \\
\text { Tien } \\
(11)\end{array}$ & $\begin{array}{l}\text { Prasad \& } \\
\text { Kulacki(1 } \\
4)\end{array}$ & $\begin{array}{l}\text { Dawo } \\
\text { od } \\
(15)\end{array}$ & $\begin{array}{l}\text { Present } \\
\text { Work }\end{array}$ \\
\cline { 2 - 7 } & 2.1 & 2.2 & 2.117 & 2.19 & 2.22 & 2.13 \\
\cline { 2 - 8 } & 3.56 & 3.6 & 3.25 & 3.4 & 3.472 & 3.48 \\
\hline
\end{tabular}

Table (1) Two-Dimensional Comparison

After this comparison the program was modified to accommodate the variation in the problem geometry and boundary conditions. This program used on different numbers of grid point to reach a constant value of the heat flow rate ratio (heat release from the hot boundaries to that gained by cold body), as shown in figure (2), and it was found that this ratio becomes constant at the value of 0.98 which is at a number of grid points 80 . This number of grid points was then used throughout the present work. 
Dawood : Numerical study of free convection heat transfer through porous.....

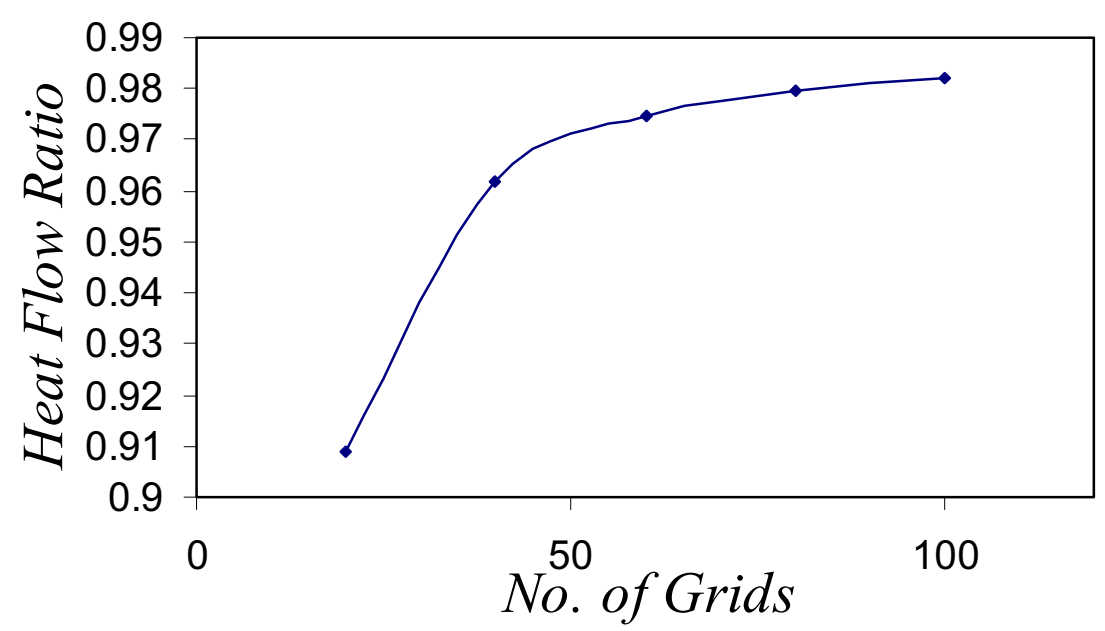

Figure(2) Relation Between Heat Flow Ratio and no. of Grids

for Body Size $1 / 2 \times 1 / 2$ and $\mathrm{Ra}=200$

\section{Flow Regime}

Before the results for various ranges of body sizes and Rayleigh numbers are presented, it is worthwhile to discuss the different flow regimes encountered in free convection in the porous enclosures.

Convection heat transfer in porous cavity is usually classified as belonging to the conduction, asymptotic, and the boundary layer flow regimes[13]. As the temperature of the hot wall begins to rise, the energy is first transported to the cold wall by pure conduction, and the Nusselt number is unity. A large temperature difference results in a moderate circulatory parallel flow. This situation is achieved either for Rayleigh number close to zero or when the distance between hot and cold wall is small and finite Ra. Any further increase in $\mathrm{Ra}$ enhances the stratification in the core, and the contribution of heat transfer by convection near the wall increases. The situation is termed as asymptotic (stratified) flow. Finally, a sufficiently large temperature difference across the cavity causes a boundary layer flow regime in which the dominant mode of heat transfer is convection.

Between the conduction regime and the boundary layer regime, two different situations can exist: one when the boundary layer effects on heat transfer are small and the other when the boundary layer effects on heat transfer are not small but conduction in the core is also not negligible. The former will continue to be as asymptotic flow whereas the latter will be referred to as the "pseudo boundary regime". The significance of this designation is that the heat transfer is mainly by convection through wall boundary layers and by conduction through the core.

\section{Results and Discussion}

\subsection{Temperature and Flow Fields:}

For pure conduction (zero Rayleigh number) the isotherms are symmetric about the horizontal $(\mathrm{y}=0.5)$ and about the vertical $(\mathrm{x}=0.5)$ centerlines. As $\mathrm{Ra}$ 
increased the constant temperature lines (Isotherms) change around the body. Most of the heat gained by the body is at the upper horizontal surface, figure $(3-\mathrm{a}, \mathrm{Ra}=100)$, where the highest vertical temperature gradient exist. A circulatory motion is established around the body because of the buoyancy effects, figure $(3-b, R a=100)$. It can been seen that the flow consists of two primary cells rotating in the counter clockwise direction at the right half of the cavity, and clockwise at the other half. Further increase in Ra to 300, causes the isotherms to change their direction. The slopes become less because of buoyancy force and high temperature difference. Temperature gradient $\partial \mathrm{T} / \partial \mathrm{y}$ at the upper surface of the body is high that means most of the heat gained by the body is at this surface.

Further more, a multicellular flow will appear, and the flow field comprises primary cells of relatively high velocity, circulating in the zone between the two vertical walls hot and cold, and secondary cells of relatively low velocity, circulating in the field below the body. A multicellular flow causes more efficient fluid mixing due to the counterrotating additional cells. Similar flow has been found in case of concentric cylinders [24], where for radius ratio $(\mathrm{R}=2)$ the flow changes from a two-cell to a four-cell regime at $\mathrm{Ra}=100$, but here it appeared at $\mathrm{Ra}=255$.

The explanation of this behavior is as follow, as Ra increased the velocity of the fluid increased due to increasing buoyancy effects and the horizontal movement of the fluid near the lower corners of the body becomes less. Under these conditions, the fluid particles that are moving down beside the vertical cold wall and near the lower corner of the body is facing a region of higher temperature. This region obliges the fluid to change its direction towards the colder region. At this point, the flow is divided into a primary and secondary cell. The flow at the secondary cells is similar to the case of free convection ina horizontal porous layer heated from below, which had been studied by many investigators [14].

As in figure 3, for large $\mathrm{Ra}(500)$ and at the core, which is located at the region between the hot and cold vertical walls, the horizontal temperature gradients are quite modest and the flow proceeds to the boundary layer regime.

Figure 4, the same number of secondary cells will appear when the body size becomes $(1 / 2 \times 1 / 2)$ they appear at $\mathrm{Ra}=135$. The velocity of the flow is higher here because the distance between the hot and cold boundaries is less. There is a conduction regime below the body before appearing the secondary flow, where the isotherms are horizontal approximately. Core region will be larger and longer in this size as shown for $\mathrm{Ra}=500$ and the flow is mainly at the boundary layer regime.

Increasing in dimensions of the body to $(2 / 3 \times 2 / 3)$ leads to appear more number of secondary cells. They are increased from two to four to six cells with increasing Ra figure (5). Most heat transfer occurs in the region below the horizontal hot and cold surfaces especially at high Ra. Core region will be smaller. 


\subsection{Heat Transfer Results:}

This section presents the effects of Rayleigh number and the body size on the relative increase in the heat transfer (Nusselt number) graphically. The Rayleigh number ranged from 0 to 500 , due to stability of the problem (no correct results can be obtained beyond this value).

Figure (6), depicts the variation of the Nusselt number as a function of the Rayleigh number. In the conduction regime, where the Nusselt number is one for all sizes of the body, this regime gets extended with increasing the body size. For body size $(1 / 4 \times 1 / 4)$, the conduction regime (onset of convective heat transfer) ends at low Ra less than 25 , whereas for size $(2 / 3 \times 2 / 3)$ it continues up to $\mathrm{Ra}=125$. In general, the slope of the Nusselt number curve is higher in the asymptotic flow regime and is lower for the boundary layer flow regime. The upper curve in the same figure, shows that the rate increase in heat transfer is moderate in the range (350-425), where the flow will be asymptotic, above $\mathrm{Ra}=425$ the slope will be high, where the most mode of fluid flow is boundary layer flow regime.

The effect of varying the isothermal body size on heat transfer are illustrated in figure(7) for $\mathrm{Ra}=100,300,500$. In general, when the body size increases the rate of heat transfer decrease. At low $\mathrm{Ra}(\mathrm{Ra}=100)$, it can be seen that there are no considerable changes in Nusselt number as the body size increased, especially above 0.4 body size, because the buoyancy force is small. The slope of curves increase at $\mathrm{Ra}=300,500$, where the circulating fluid in the primary and secondary cells effect on the amount of heat transfer.

\section{Conclusions}

1. From the results, it has been demonstrated that the average Nusselt number is a strong function of Rayleigh number and body size. For fixed Rayleigh number the Nusselt number increased as the body size decreased, whereas for fixed body size as the Rayleigh number increased the relative convective heat transfer increased.

2. It is observed that a large fraction of the heat is transferred within the lower horizontal surfaces of body and cavity.

3. A multicellular flow has been observed at different Ra depending on body size. The flow structure comprises to a primary re-circulating cells of relatively high velocity on the sides of the body, and secondary cells of relatively low velocity appearing in the region below the body.The secondary cells increasing the fluid mixing. 

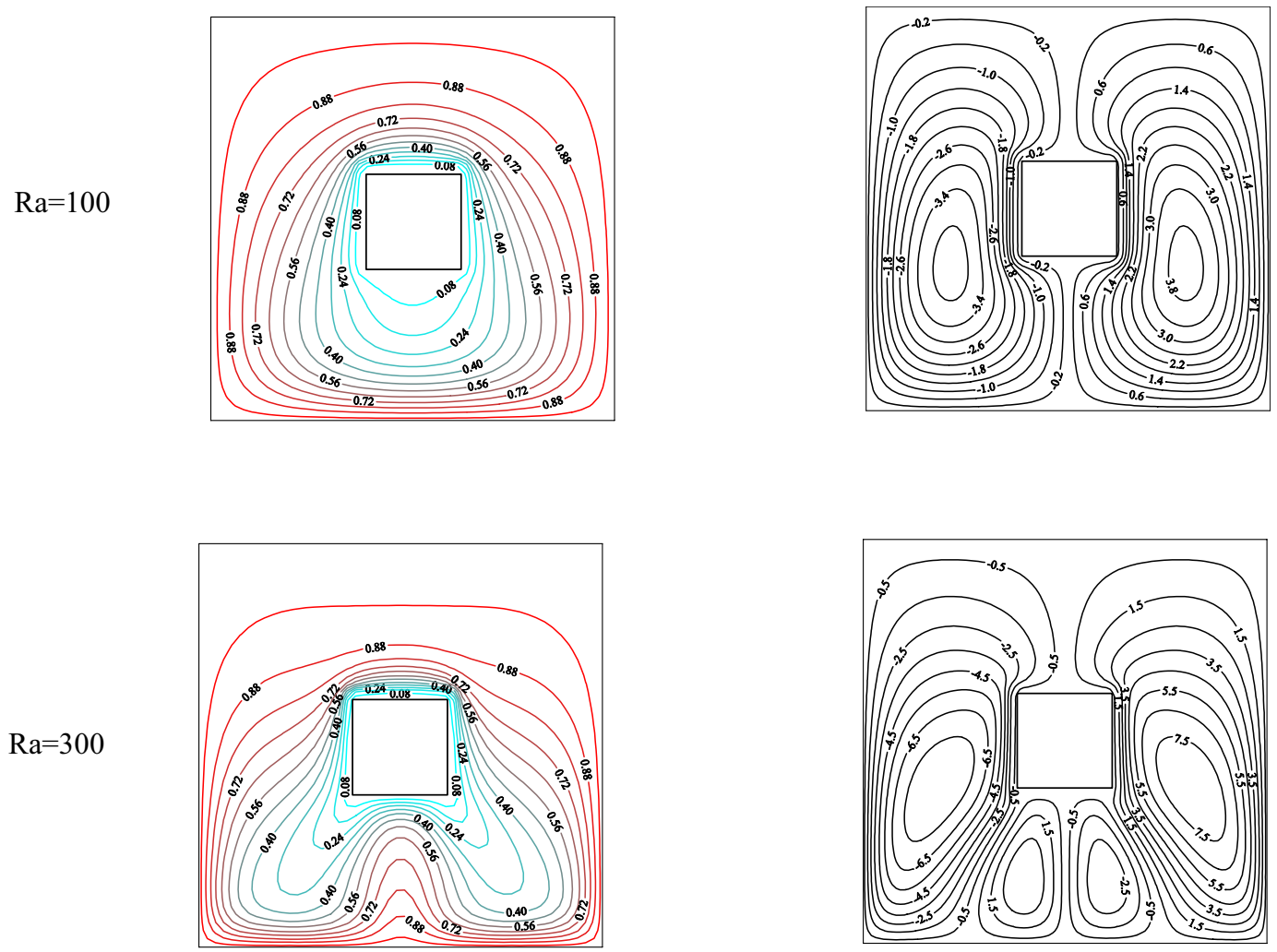

$\mathrm{Ra}=500$

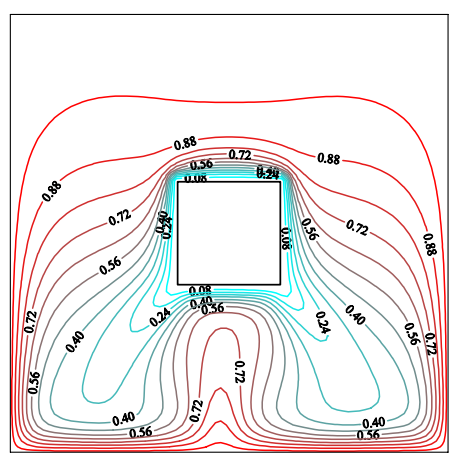

(a)

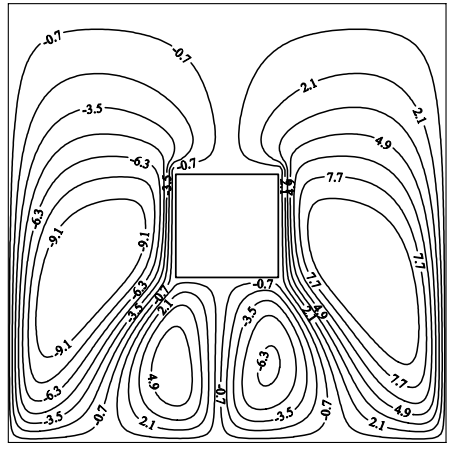

(b)

Figure ( 3 ) (a ) Isotherms, ( b ) Streamlines at body size ( 1/4x1/4 )and different rayleigh number 
Dawood : Numerical study of free convection heat transfer through porous.....
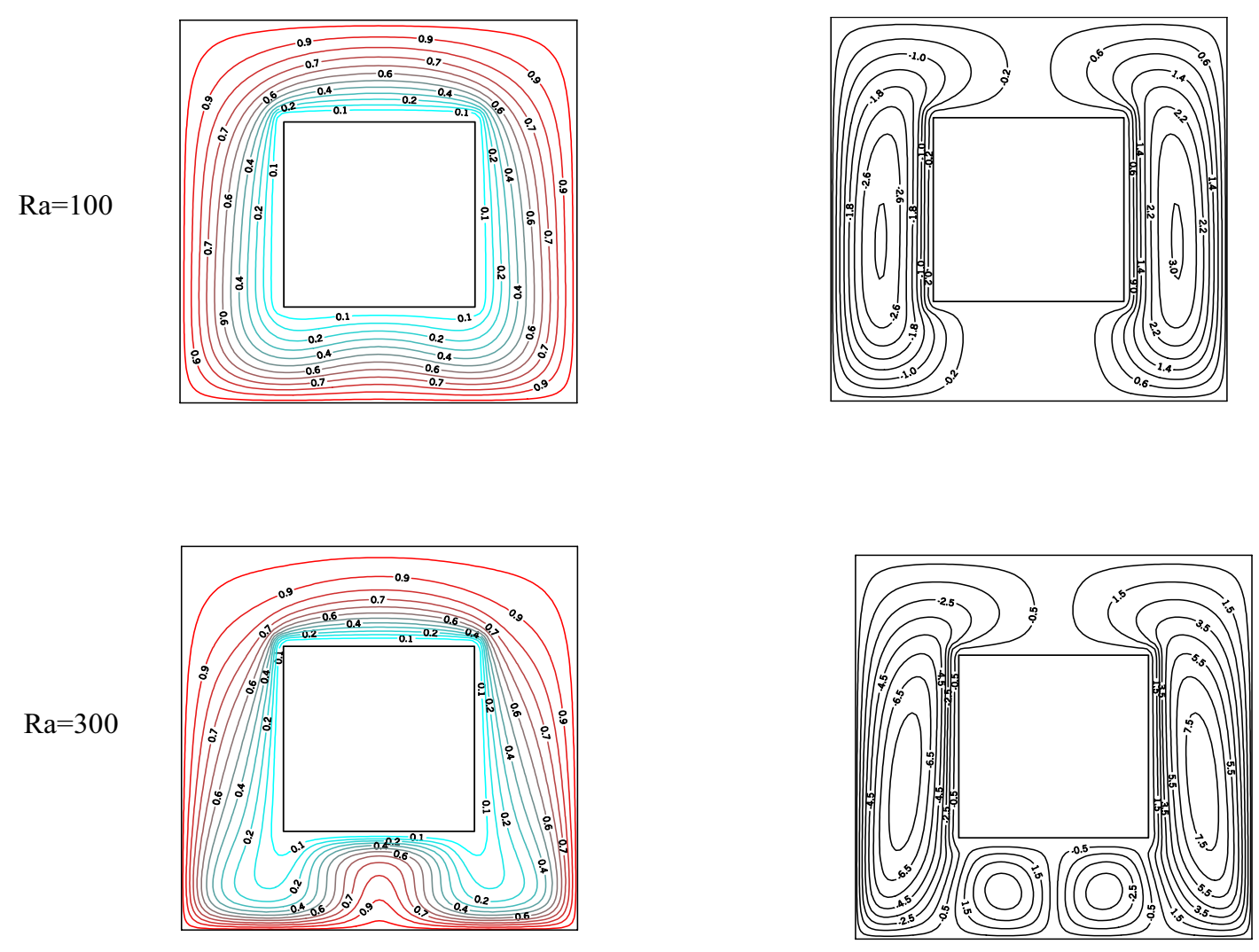

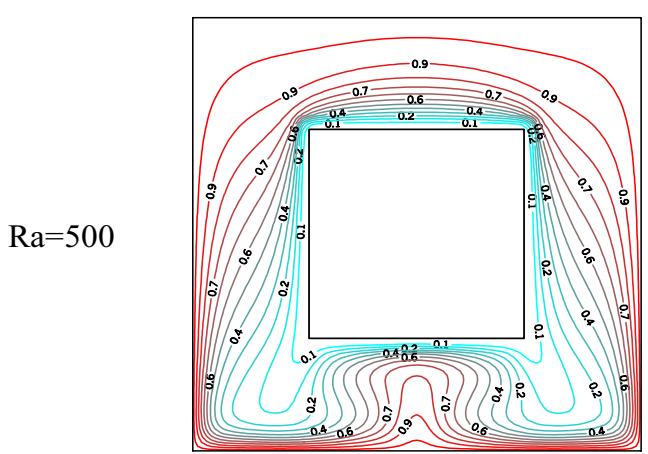

(a)

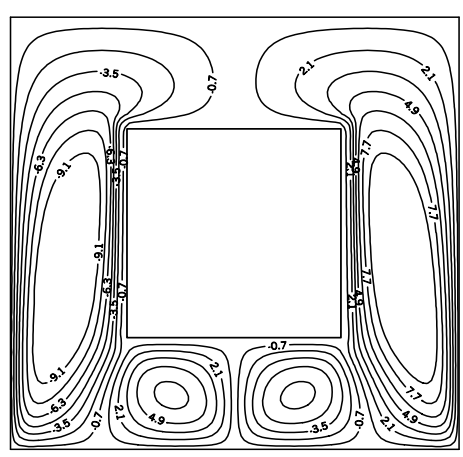

(b)

Figure ( 4 ) (a ) Isotherms, ( b ) Streamlines at body size ( 1/2x1/2 )and different rayleigh number 
$\mathrm{Ra}=100$
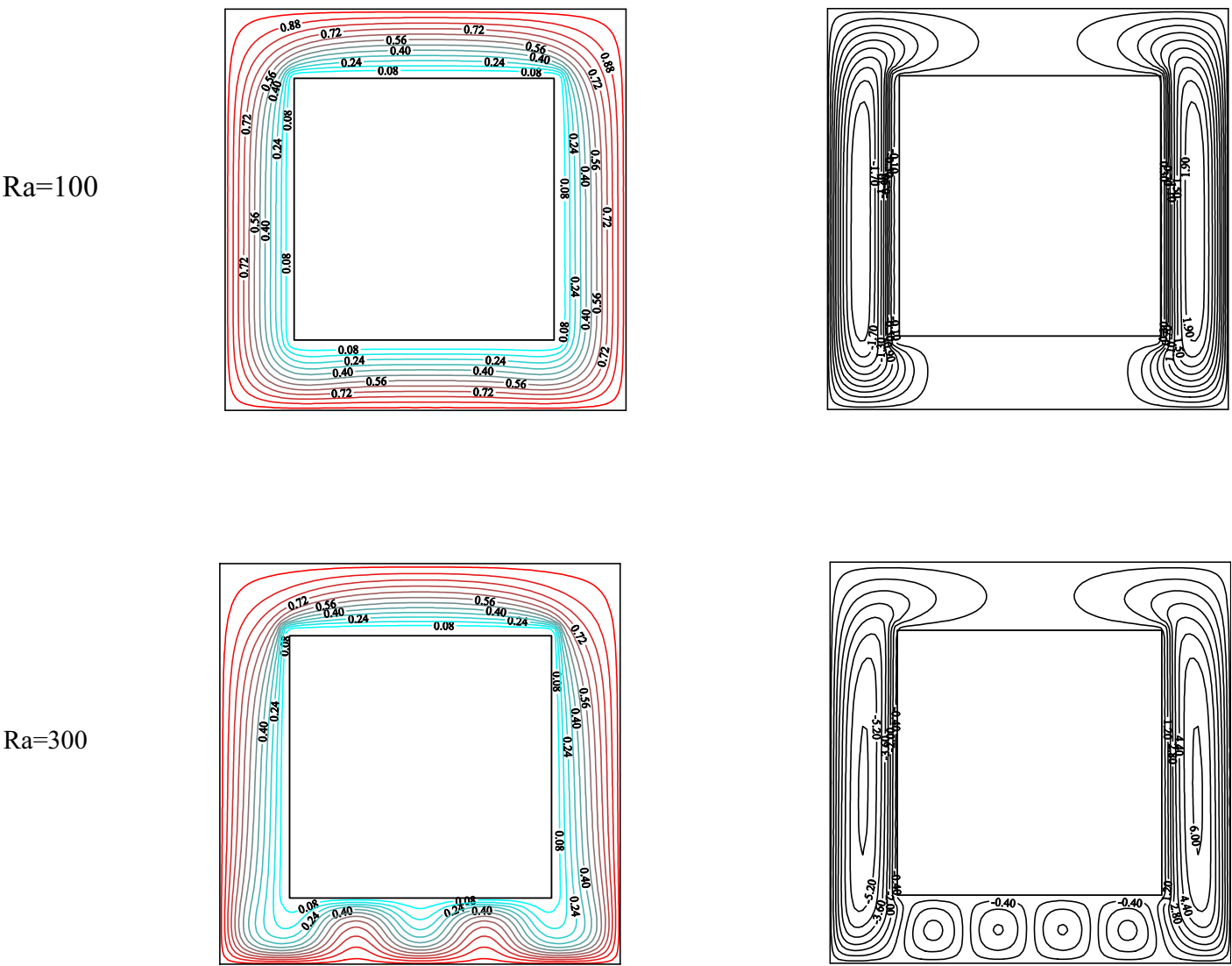

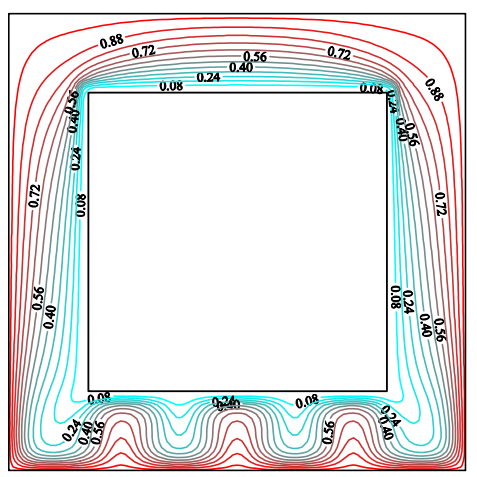

(a)

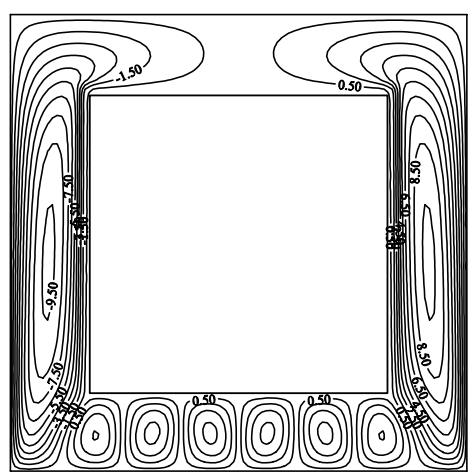

(b)

Figure ( 5 ) (a ) Isotherms, ( b ) Streamlines at body size (2/3x2/3) and different rayleigh number 
Dawood : Numerical study of free convection heat transfer through porous......

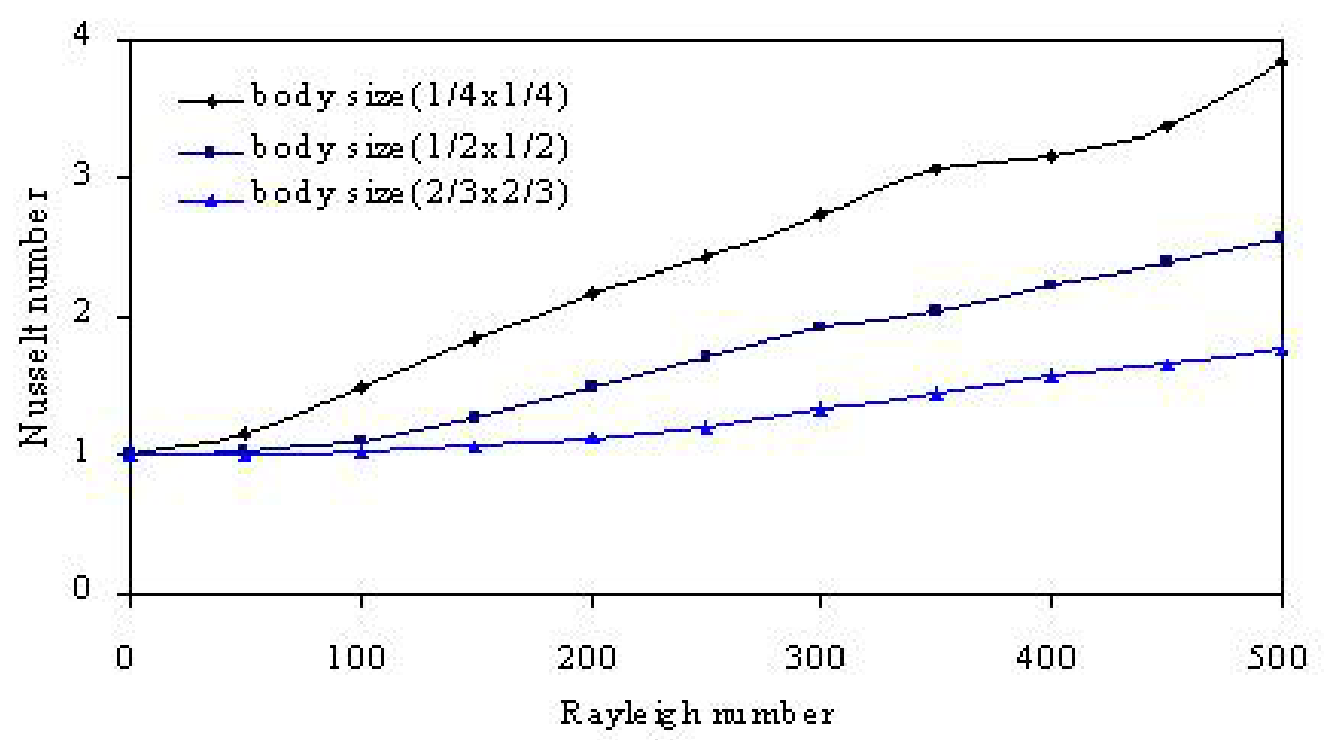

Figure ( 6 ) Relation Between Rayleigh no. and Nusselt no. for Different Body Size

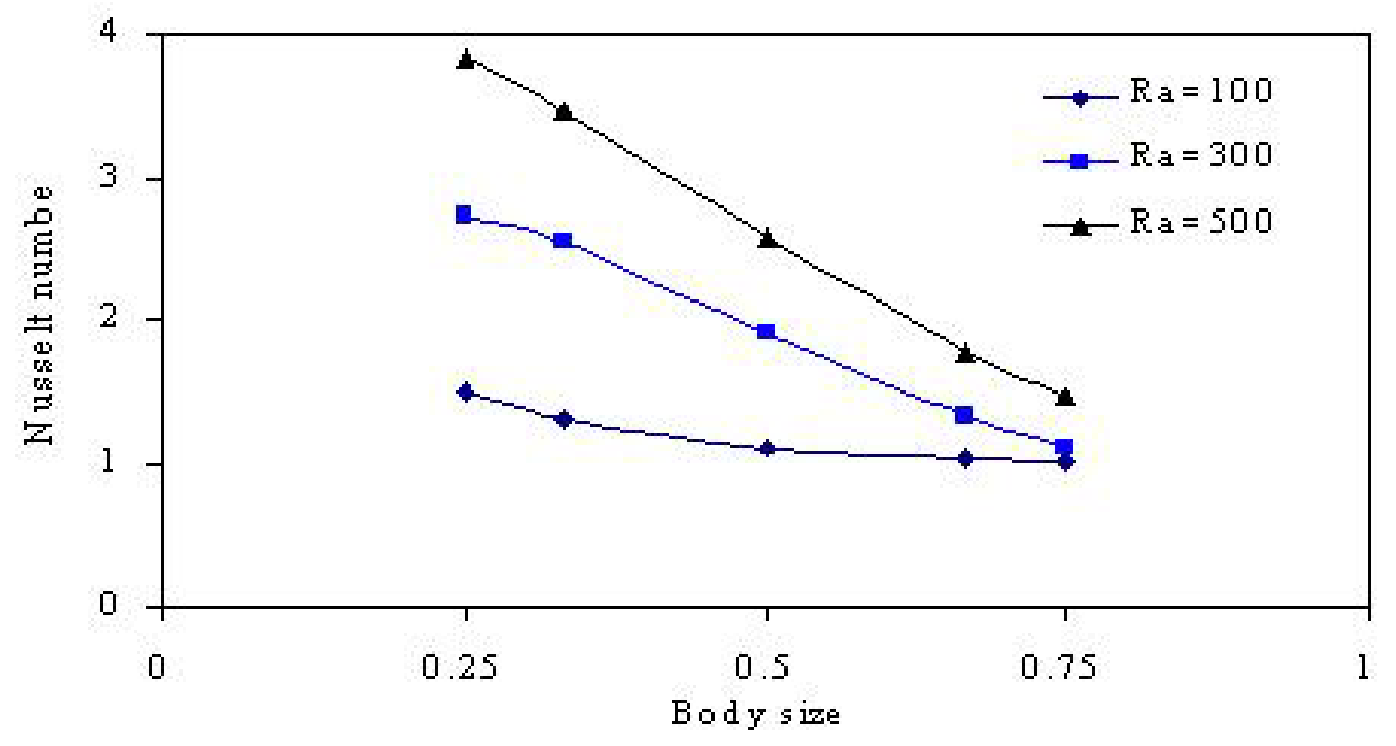

Figure ( 7 ) Relation Between Body Size and Nusselt Number for Different Ra 


\section{References:}

1. Hori, I. Ene and Polisevski, D., Thermal Flow in Porous Media, D. Reidel Publishing Company, Dordrechi, Holand, 1987.

2. Horton, C.W. and Rogers, F.T., "Convection Current in a Porous Medium," J. App. Phys. ,16 ,pp. 367-370, 1945.

3. Nobuhiro, S. ,Shoichiro, F. ,and Hideo, I. ,"Heat Transfer in a Confined Rectangular Cavity Packed with Porous Media," Int. J. Heat Mass Transfer, 21, pp. 985-989, 1978.

4. Prasad, V. and Kulacki, F. A. , "Convective Heat Tranfer in a Rectangular Porous Cavity-Effect of Aspect Ratio on Flow Structure and Heat Transfer", J. Heat Transfer, 106, pp.158-165, 1984.

5. Oothuizen, P.H., "Mixed Convective Heat Transfer from a Heated Horizontal Plate in aPorous Medium Near an Impermeable Surface," J. Heat Transfer, 110, pp.390-394, 1988.

6. Arnold, F., "Natural Convection in a Porous Medium Between Concentric Horizontal Cylinders," Numerical Methods in Thermal Problems, 7, Part2, pp. 1081-1091, 1991.

7. Barbosa Mota, J.P. and Saatdjian, E., "Natural Convection in a Porous, Horizontal Cylinderical Annulas," J. Heat Transfer, 116, pp.621-626, 1994.

8. Bejan, A., Convection Heat Transfer, Wiley-Intersscience Publication, John Wiley \& Sons, Inc., 1984.

9. Carslaw, H.S. and Jaeger, J.C., Conduction of Heat in Solids, $2^{\text {nd }}$ edition, Oxford University Press, 1959.

10. Chan, B.K.C., Ivey, C.M., and Barry, J.M., "Natural Convection in Enclosed Porous Media with Rectangular Boundaries," J. Heat Transfer, 2, pp.21-27, 1970.

11. Burns, P.J., Chow, L.C. and Tien, C.L., "Convection in Vertical Slot Filled with Porous Insulation,” Int.J. Heat Transfer, 20, pp.919-926, 1977.

12. Bejan, A. and Tien, C.L., "Natural Convection in Horizontal Porous Medium Subjected to an End-to-End Temperature Difference," J. Heat Transfer, 100, pp.191198, 1978.

13. Chang, P., Handbook of Heat Transfer Applications, ed. W.M. Rohsenow, J.P. Hartnett and E. Ganic, $2^{\text {nd }}$ edition, McGraw-Hill, New York, 1985.

14. Cheng, P., "Heat Transfer in Geathermal Systems," Adv. Heat Transfer, 14, pp. 1-105, 1978.

15. Dawood A. S.," Steady Three-Dimensional natural Convection in Porous Media Via Multigrid Method," Ph. D. Dissertation, Colorado State University, 1991. 